\title{
Transversely Compact Single-Ended and Balanced Bandpass Filters with Source-Load-Coupled Spurlines
}

\author{
Fang Yan ${ }^{1, *}$, Yong Mao Huang ${ }^{2}{ }^{(0)}$, Tao Huang ${ }^{2}$, Shuai Ding ${ }^{3}{ }^{(0)}$, Kenian Wang ${ }^{1}$ \\ and Maurizio Bozzi 4 (D) \\ 1 Center of Airworthiness, Civil Aviation University of China, Tianjin 300300, China; wangkenian@126.com \\ 2 School of Electrical and Electronic Information, Xihua University, Chengdu 610039, China; \\ ymhuang128@gmail.com (Y.M.H.); huang1987tao@yeah.net (T.H.) \\ 3 Institute of Applied Physics, and School of Physical Electronics, University of Electronic Science and \\ Technology of China, Chengdu 610054, China; uestcding@hotmail.com \\ 4 Department of Electrical, Computers and Biomedical Engineering, University of Pavia, 27100 Pavia, Italy; \\ maurizio.bozzi@unipv.it \\ * Correspondence: fyan@cauc.edu.cn; Tel.: +86-158-229-69896
}

Received: 18 March 2019; Accepted: 6 April 2019; Published: 10 April 2019

\begin{abstract}
Multi-function wireless systems demand multi-channel transmit/receive (TR) modules, particularly as multiple functions are required to operate simultaneously. In each channel, passive components, including bandpass filters, must be compact, or at least transversely compact; thus, the entire circuitry of the channel will be slender, and consequently multiple channels can be parallel-arranged conveniently. In this work, single-ended and balanced bandpass filters for multi-channel applications are presented. As a unique resonator, the U-shaped stepped impedance resonator (USIR) can achieve size miniaturization compared with its corresponding uniform impedance resonator (UIR) counterpart. Hence, with the utilization of USIRs, the proposed bandpass filters are able to acquire compact transverse sizes. Moreover, by using the source-load coupling scheme, two transmission zeros (TZs) are respectively generated at the lower and upper sides of the passbands, which is useful for improvement of the selectivity performance. In addition, spurlines are introduced at the input and output ports to produce another TZ to further enhance the stopband performance, which cannot be acquired by the UIR or stepped impedance resonator (SIR). To verify the aforementioned idea, one single-ended and one balanced bandpass filter are implemented, with experimental results in good agreement with the corresponding simulations. Meanwhile, as compared with some similar works, the proposed balanced filter achieves compact transverse size, sharp selectivity skirt, and wide stopbands up to the fourth-order harmonic with suppression over $20 \mathrm{~dB}$, which illustrates its suitability for differential signal transmission application in microwave circuits and systems.
\end{abstract}

Keywords: balanced bandpass filter; common mode suppression; spurline; source-load coupling; stepped impedance resonator (SIR)

\section{Introduction}

Recently, different kinds of radio frequency (RF) and microwave systems for various wireless applications like commutation, radars, and sensors have been widely investigated. As applications become increasingly concrete and specific, more and more functions are expected to be integrated into a single system, and the system circuitry eventually gets more and more complex. Meanwhile, there is also an increasing demand on high-performance passive components, to ensure that multiple 
functions in the single system can all operate well. Passive components including filters with excellent performance in amplitude noise, phase jitter, spurs, and harmonics are highly desired, particularly for applications such as fifth generation (5G) mobile access and transmission, high-resolution imaging radar, automotive anti-collision sensors, and many other specific applications. For instance, Figure 1 gives the diagram of a typical multi-function wireless system with multi-channel receivers. It can be easily obtained that various kinds of active and passive components, including antennae, low noise amplifiers (LNAs), mixers, power amplifiers (PAs), and filters, are contained in each channel (frequency synthesizers for carriers are omitted). On one hand, as depicted in [1], active components are mostly integrated circuits with extremely compact sizes. Hence, the passive components of a multi-channel radio typically occupy about $65 \%$ of its circuitry size. Therefore, size reduction of passive components is important to miniaturize the overall size of the system. On the other hand, for the receiver of each channel in Figure 1, all components, especially the passive ones, are supposed to be compact (or at least transversely compact) therefore the entire channel circuitry is slender. In this way, all channels can be parallel-arranged easily and the circuit board of the multi-channel system will eventually be compact. Thus, passive components with compact transverse size are quite useful for multi-channel systems.

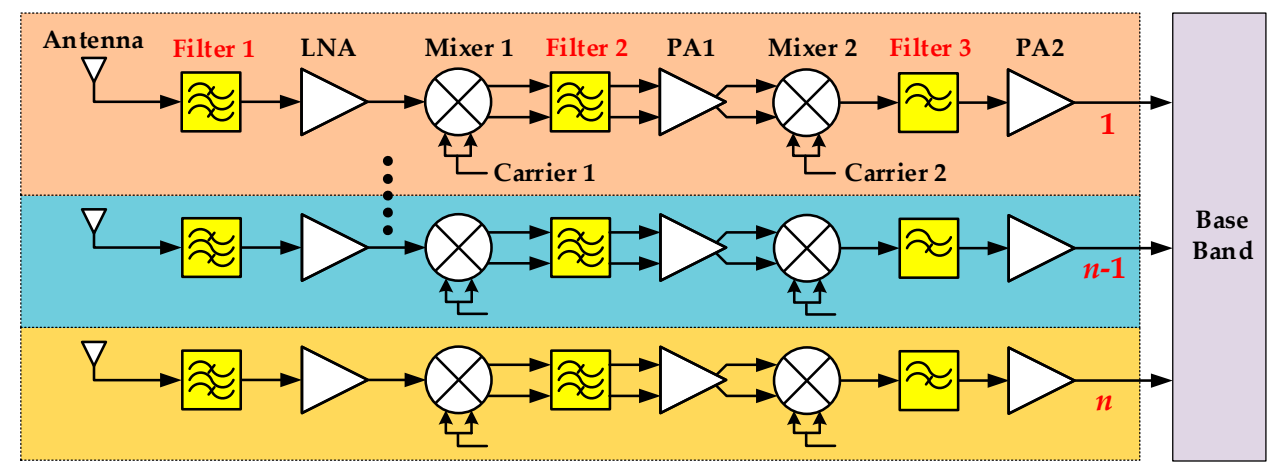

Figure 1. Diagram of a typical multi-function wireless system with multi-channel receivers.

Meanwhile, it can be inferred from Figure 1 that most of the passive components in the multi-channel system are filters. For instance, as shown in Figure 1, Filter 1 is a single-ended bandpass filter, Filter 2 is a balanced one (which is also called a differential filter) and Filter 3 is a single-ended lowpass filter. Actually, passband selecting by filter seems be one of the most effective approaches for suppression of the unwanted spurs or harmonics in the received signals. In the literature, guided-wave structure of different forms, planar and nonplanar, have been developed to implement high-performance filters. It is known that microstrip technology is conventional but has the advantage of being able to integrate with other planar components and circuits due of its simple and compact structure [2]. Compared to the waveguide, substrate-integrated waveguide, and coplanar waveguide technologies, it is very easy to design a variety of filters with complex structures and coupling mechanisms as well as miniature size by using microstrip line. In past decades, microstrip uniform impedance resonators (UIRs) of various electrical lengths have been widely utilized to design filters [2]. To improve the design flexibility of UIRs and miniaturize their size, the stepped impedance resonator (SIR) has been developed [3]. Its modified forms-folded SIR (FSIR) and U-shaped SIR (USIR)—can achieve further size reduction [4]. On the other hand, some interesting techniques, such as defected ground structure (DGS), defected microstrip structure (DMS), spurline, and source-load coupling, have been proposed to improve the performance of microstrip filters [5-7]. In [8], both the SIR and spurline are utilized to develop a balanced filter with wide stopband. However, its superconducting process limits its application in practical RF systems. Later, the spurline-stub resonator is used to suppress the even-mode noise of differential filter. However, it needs to undergo micromachining fabrication and wire-bonding processes, which are complex [9]. A dual-band balanced filter based on open-ended half-wavelength UIR resonator and interdigital capacitor is proposed in [10], while another case was made of a short-ended half-wavelength UIR 
resonator with multilayer structure [11]. In [12], the aggressive space mapping method is utilized to synthesize a balanced filter incorporating the SIR, which achieves an excellent common mode rejection ratio (CMRR) about $50 \mathrm{~dB}$. However, since the filter contains seven SIRs, its physical size is bulky. Here, it is essential to declare that the CMRR in this work is defined as usual, as CMRR = $\left|S_{\mathrm{dd} 21}\right| /\left|S_{\mathrm{cc} 21}\right|$. On the other hand, the matrix transformation method is employed to synthesize the balanced filter with common mode (CM) noise absorption, which seems to be an effective strategy [13]. On the other hand, selectivity and stopband performance of the synthesized filter are not good owing to its impedance-transformation-based structure. The dumbbell-shaped resonator is etched on the ground plane of the differential filter to improve its CMRR to $57 \mathrm{~dB}$ [14]. Recently, both the SIRs and interdigital capacitors are utilized together to produce two TZs for the balanced filter in [15]. In addition, some multi-function balanced filters are also investigated. In [16], a SIR-based diplexer with high performance is reported. The multi-stub-loaded transmission line was included to realize quasi-reflectionless single- and dual-band balanced filters [17]. Another work even integrates power dividing function in the single-ended-to-balanced filter with wideband CM suppression [18].

Most of the aforementioned works exhibit high performance in CMRR, stopband, or selectivity. However, these high performances are mainly achieved at the cost of large physical size, which is not proper for system integration and packaging applications. It is known that for system integration and packaging applications, both the physical size and stopband performance, as well as selectivity, are significant. In this work, a single-ended and a balanced filter are investigated, taking account of size, selectivity, and stopband simultaneously. The USIR is utilized as basic resonator for size reduction while the source-load coupling topology is able to produce two TZs for selectivity performance, and spurlines included at the source and load node can generate an extra TZ to enhance the stopband performance. This work is organized as below: Section 2 introduces the USIR and spurline in detail. Sections 3 and 4 investigate implementation of the single-ended and balanced filters, respectively. In Section 5, a brief discussion is sketched, with a conclusion given at the end.

\section{Basic Elements of the Proposed Filters}

\subsection{Half-Wavelength U-Shaped Stepped Impedance Resonator}

Figure 2a gives geometries and corresponding equivalent-circuit models of the half-wavelength UIR, SIR, and USIR. As is shown, the UIR with the characteristic impedance of $Z_{0}$ can be modeled as a parallel network of inductance $L_{0}$ and capacitance $C_{0}$. The SIR consists of one high-impedance section in the center and two low-impedance sections on both sides. The high-impedance section has characteristic impedance of $Z_{\mathrm{H}}$ and electrical length of $\theta_{\mathrm{H}}$, while values are $Z_{\mathrm{L}}$ and $\theta_{\mathrm{L}}$ for the low-impedance case. The effective inductance and capacitance of the SIR can be represented by $L_{S}$ and $C_{\mathrm{s}}$. For the USIR, effective inductance and capacitance are denoted by $L_{\mathrm{u}}$ and $C_{\mathrm{u}}$. Additionally, as listed in Figure 2a, folding the straight SIR into a U shape will generate extra strip-to-strip capacitive effect between the two parallel sections and can be modeled as $C_{\mathrm{e}}$. Firstly, as the half-wavelength USIR operates at the dominant resonant mode, the electric field will distribute symmetrically along the transverse centerline. Hence, under the dominant resonant mode, the transverse centerline can be regarded as the short-circuited plane $[3,19]$. Then, the half-wavelength USIR can be equivalent to the quarter-wavelength USIR, as shown in Figure 2a. Then, resonance properties of the half-wavelength USIR can be captured from the corresponding properties of the quarter-wavelength USIR. Therefore, the input admittance of the USIR in Figure 2a (i.e., $Y_{\text {in }}$ ) is given as:

$$
Y_{\text {in }}=\frac{1}{Z_{\text {in }}}=\frac{Z_{\mathrm{L}}-Z_{\mathrm{H}} \tan \theta_{\mathrm{L}} \tan \frac{\theta_{\mathrm{H}}}{2}}{j Z_{\mathrm{L}}\left(Z_{\mathrm{H}} \tan \frac{\theta_{\mathrm{H}}}{2}+Z_{\mathrm{L}} \tan \theta_{\mathrm{L}}\right)}
$$

The USIR operates at the dominant resonant frequency, $Y_{\text {in }}=0$, which means:

$$
\mathrm{Z}_{\mathrm{L}}-\mathrm{Z}_{\mathrm{H}} \tan \theta_{\mathrm{L}} \tan \frac{\theta_{\mathrm{H}}}{2}=0 .
$$


Thus, it can be captured that:

$$
\theta_{\mathrm{H}}=2 \arctan \frac{Z_{\mathrm{L}}}{Z_{\mathrm{H}} \tan \theta_{\mathrm{L}}}
$$

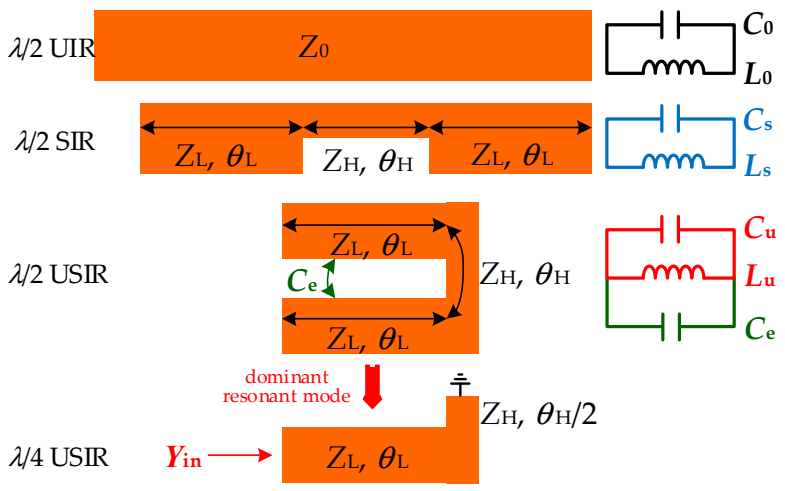

(a)

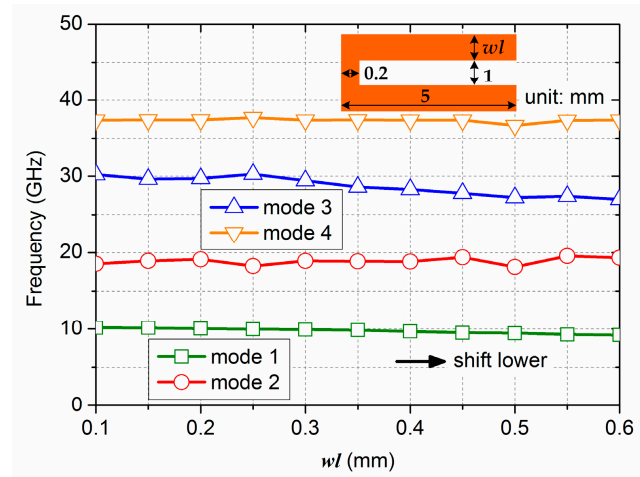

(b)

Figure 2. Geometry and resonant frequency of the U-shaped stepped impedance resonator (USIR): (a) Geometries and equivalent-circuit models of the uniform impedance resonator (UIR), stepped impedance resonator (SIR), and USIR; (b) resonant frequencies of the lowest four modes in the USIR versus width of the low-impedance section.

Then, electrical lengths of the half-wavelength USIR, $\theta_{\text {half }}$, can be calculated as:

$$
\theta_{\text {half }}=2 \theta_{\mathrm{L}}+\theta_{\mathrm{H}}=2 \theta_{\mathrm{L}}+2 \arctan \frac{Z_{\mathrm{L}}}{Z_{\mathrm{H}} \tan \theta_{\mathrm{L}}}=2 \arctan \left[\tan \theta_{\mathrm{L}}\right]+2 \arctan \frac{Z_{\mathrm{L}}}{Z_{\mathrm{H}} \tan \theta_{\mathrm{L}}} .
$$

By considering the basic properties of the arctangent function and Equation (4) together, the following can be derived: as $Z_{\mathrm{L}}=Z_{\mathrm{H}}, \theta_{\text {half }}$ is equal to $\pi$, which is the very electrical length of the conventional half-wavelength UIR under the dominant resonant mode; as $Z_{\mathrm{L}}>Z_{\mathrm{H}}, \theta_{\text {half }}$ is able to larger than $\pi$; as $Z_{\mathrm{L}}<Z_{\mathrm{H}}, \theta_{\text {half }}$ can be smaller than $\pi$. Therefore, by selecting the value of $Z_{\mathrm{L}}$ to be smaller than that of $Z_{\mathrm{H}}$, the electrical length of USIR can be smaller than that of the conventional UIR, which means the physical length of USIR is able to be shorter than that of the conventional UIR, and eventually size reduction of the resonator will be achieved.

Secondly, as depicted in [2,3], the dominant resonant frequencies of UIR, SIR, and USIR in Figure 2a can be expressed as:

$$
\begin{gathered}
f_{0}=\frac{1}{2 \pi \sqrt{L_{0} C_{0}}}, \\
f_{\mathrm{s}}=\frac{1}{2 \pi \sqrt{L_{\mathrm{s}} C_{\mathrm{s}}}}, \\
f_{\mathrm{u}}=\frac{1}{2 \pi \sqrt{\overline{L_{\mathrm{u}}\left(C_{\mathrm{u}}+C_{\mathrm{e}}\right)}} .} .
\end{gathered}
$$

Generally, since the extra strip-to-strip capacitive effect produced by the folding is much weaker than the inherent inner capacitive effect of USIR, it can be obtained that $C_{\mathrm{e}}<<C_{\mathrm{u}}$. Hence, for the simplification of analyses, the parasitic strip-to-strip capacitive effect of the USIR can be neglected and its dominant resonant frequency can be calculated as:

$$
f_{\mathrm{u}} \approx \frac{1}{2 \pi \sqrt{L_{\mathrm{u}} C_{\mathrm{u}}}} .
$$

Subsequently, distribution of the higher-order modes is important for the transmission property of the resonator. To study the property of higher-order modes of the USIR directly, some numerical simulations are carried out using a commercial three-dimensional full-wave simulator. The substrate 
for simulation is set with a thickness of $0.508 \mathrm{~mm}$, a relative permittivity of 2.2, a loss tangent of 0.001 , and a copper cover of $0.035 \mathrm{~mm}$. Figure $2 \mathrm{~b}$ lists the simulated resonant frequencies of the lowest four modes in the USIR versus width of the low-impedance section, with some fixed geometrical parameters of the USIR being given in the inset as well. From Figure $2 b$, it can be obtained that the resonant frequencies of modes 2, 3, and 4 are about the second-, third-, and fourth-order harmonics of mode 1 (the dominant resonant mode) of the USIR. Thus, USIR is a relatively simple resonator and can be utilized easily, as no complex parasitic modes exist. Furthermore, as the width of the low-impedance section $w l$ gets wider, the dominant resonant frequency of the USIR becomes lower. That is, the smaller the impedance ratio $Z_{\mathrm{L}} / Z_{\mathrm{H}}$ is, the lower the dominant resonant frequency will be. Therefore, a smaller impedance ratio can be helpful for size reduction of the resonator and other related components. Meanwhile, Figure 3 shows electric field distributions of the lowest four modes in the USIR. For these simulations, the value of $w l$ is $0.4 \mathrm{~mm}$.
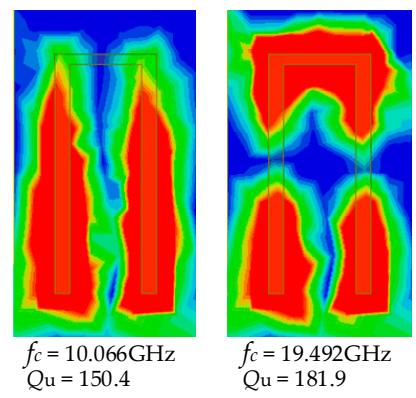

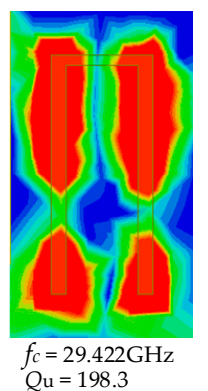

\begin{tabular}{l}
$\mathrm{Qu}_{\mathrm{u}}=198.3$ \\
\hline
\end{tabular}
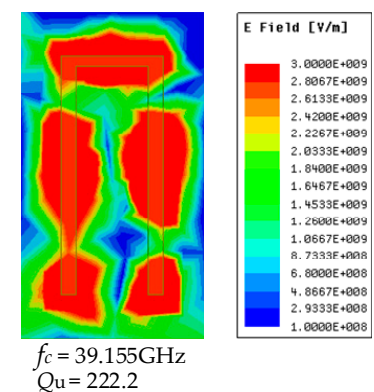

Figure 3. Electric field distributions of the lowest four modes in the USIR.

\subsection{T-Shaped Spurline}

As mentioned in Section 1, defected ground structure (DGS), defected microstrip structure (DMS), and spurline are advanced techniques for improvement of the stopband performance of microstrip filters. Figure 4 gives derivation from the conventional microstrip transmission line to the DGS, DMS, and various spurlines. As depicted in [5,6], the DGS, DMS, and quarter-wavelength spurline can all be equivalent to a parallel network of resistance, inductance, and capacitance (RLC). In practical design, this parallel network is cascaded into the component and can produce a $\mathrm{TZ}$ at a specific frequency. Location of the TZ is mainly determined by physical size of the defected structure in DGS, DMS, and spurline. In the literature, various modified spurlines, including the mender spurline and multi-spurline, have been developed. As discussed in [6], the mender has a longer slot compared with the conventional spurline (which has straight slots), which will provide a larger product of equivalent capacitance and inductance to generate a TZ at lower frequency. For filter cases, generating a TZ at lower frequency of the upper stopband usually means improving the selectivity performance. On the other hand, it can be seen from Figure 4 that the multi-spurline has multiple slots of different lengths, which will produce multiple TZs at different frequencies and be helpful for improvement of stopband performance of filters.

In this work, the T-shaped spurline is proposed, with its geometry shown in Figure 4. Obviously, compared with the conventional spurline, the proposed one contains more flexible dimensions, since the T-shaped slot includes three sections of various widths and lengths. The proposed T-shaped spurline can be equivalent to a parallel RLC network as well, with the lumped elements being given as [6]:

$$
\begin{gathered}
R_{\mathrm{T}}=\left.2 Z_{0}\left(\frac{1}{\left|S_{21}\right|}-1\right)\right|_{f=f_{0}}{ }^{\prime} \\
C_{\mathrm{T}}=\frac{\sqrt{0.5\left(R_{\mathrm{T}}+2 Z_{0}\right)^{2}-4 Z_{0}^{2}}}{2.83 \pi Z_{0} R_{\mathrm{T}} \Delta f},
\end{gathered}
$$




$$
L_{\mathrm{T}}=\frac{1}{\left(4 \pi f_{0}\right)^{2} C_{\mathrm{T}}}
$$

where $L_{\mathrm{T}}, C_{\mathrm{T}}$, and $R_{\mathrm{T}}$ are the equivalent inductance, capacitance, and resistance, respectively, of the proposed T-shaped spurline. $\left|S_{21}\right|$ is the insertion loss. $f_{0}$ is the resonant frequency (frequency of the TZ) and $\Delta f$ is the $3 \mathrm{~dB}$ bandwidth of $\left|\mathrm{S}_{21}\right| . Z_{0}$ is the characteristic impedance of the transmission line, which is typically set as $50 \mathrm{ohm}$. In the specific implementation, values of $L_{\mathrm{T}}, C_{\mathrm{T}}$, and $R_{\mathrm{T}}$ can be calculated by considering Equations (9)-(11), incorporating the specified design frequency of the TZ and numerical simulation results of the T-shaped spurline.

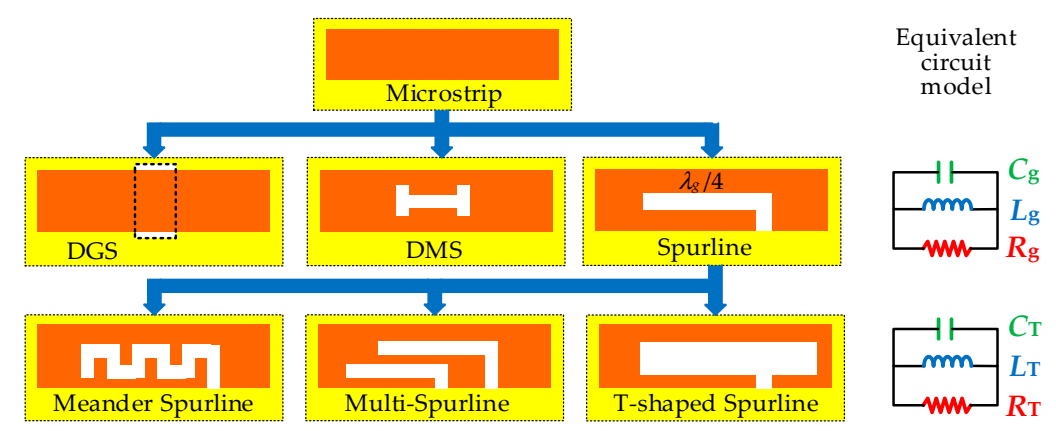

Figure 4. Derivation from the conventional microstrip to the defected ground structure (DGS), defected microstrip structure (DMS), and various spurlines.

\section{Single-Ended Bandpass Filter with Source-Load-Coupled Spurlines}

To demonstrate the effectiveness of the USIR and proposed T-shaped spurline, a single-ended bandpass filter is designed. First of all, as mentioned above, the source-load coupling scheme is able to improve performance of the filter notably. Hence, the source-load coupling scheme is employed in the proposed single-ended filter design as well. Figure 5 sketches the detailed configuration and coupling scheme of the proposed single-ended filter based on the USIR and T-shaped spurline. Firstly, as shown in Figure 5a, the proposed single-ended bandpass filter contains two USIRs, two spurlines, two 50 ohm microstrip lines at the input and output ports, and two tapered transitions between the spurlines and $50 \mathrm{ohm}$ microstrips. Secondly, as shown in Figure 5b, the filter can generate a major coupling path as S-R1-R2-L. Here, S and L represent the source and load nodes, respectively. R1 and R2 denote resonator nodes. Meanwhile, in the proposed single-ended filter, there is another coupling path (i.e., $\mathrm{S}-\mathrm{L}$ ) which is mainly contributed to by the source-load coupling scheme. With the two aforementioned coupling paths operating together, the cross-coupling will be generated, so that two TZs, separately located at the lower and upper sides of the passband, can be generated, which will help to enhance the selectivity of the filter remarkably. Thirdly, the source and load nodes mainly consist of the proposed T-shaped spurlines, thereby an extra transmission zero can be produced at the upper stopband to extend the bandwidth of the stopband of the proposed single-ended filter.

Thereafter, as introduced in [2], operation frequency of the filter is mainly determined by the sizes of the employed resonators, whereas its bandwidth is mainly controlled by the coupling coefficients between adjacent resonators. Furthermore, tapered transitions will also influence both the central frequency and the bandwidth, since they can affect the value of the external quality factor notably. To study the influence from the USIR on the operation frequency of the filter more clearly, some numerical simulations have been done on the same aforementioned substrate by using the same commercial electromagnetic simulator used above. In these simulations, some fixed geometrical parameters are: $w s=1.52, l t=1.5, a 0=1.2, b 0=2.0, a 1=0.6, b 1=6.0, c 1=2.0, v 1=0.5, s 1=0.5, s 2=0.3$, $s 3=0.3$ (units: $\mathrm{mm}$ ). Figure 6 shows simulated IS21 $\mid$ of the proposed single-ended filter with the variation of some geometrical parameters. For Figure $6 \mathrm{a}, b 3=2.2 \mathrm{~mm}, a 2=0.4 \mathrm{~mm}, a 3=0.2 \mathrm{~mm}$. As $b 2$ increases from $3.9 \mathrm{~mm}$ to $4.5 \mathrm{~mm}$, both the passband and the first two TZs shift lower, while the highest TZ only shifts slightly lower. For Figure $6 \mathrm{~b}, b 2=4.2 \mathrm{~mm}, a 2=0.4 \mathrm{~mm}, a 3=0.2 \mathrm{~mm}$. In this case, 
with a longer $b 3$, the passband, TZ1, and TZ2 all shift lower, whereas TZ3 remains nearly unchanged. In Figure $6 \mathrm{c}, b 2=4.2 \mathrm{~mm}, b 3=2.2 \mathrm{~mm}, a 3=0.2 \mathrm{~mm}$. For this case, the passband, TZ1, and TZ2 all shift lower with the increasing of $a 2$, while TZ3 is also nearly unmoved. Finally, for Figure $6 \mathrm{~d}, b 2=4.2 \mathrm{~mm}$, $b 3=2.2 \mathrm{~mm}, a 2=0.3 \mathrm{~mm}$. From Figure $6 \mathrm{~d}$, the wider $a 3$ is, the lower the passband is. Moreover, TZ1 and TZ2 will shift much lower than TZ3 as $a 3$ gets wider.

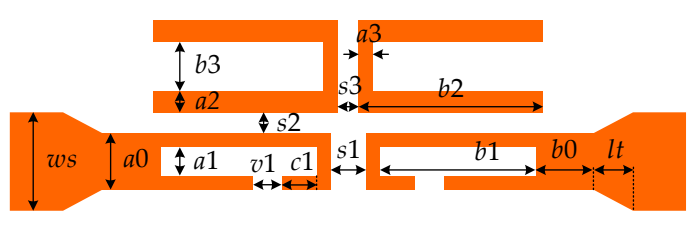

(a)

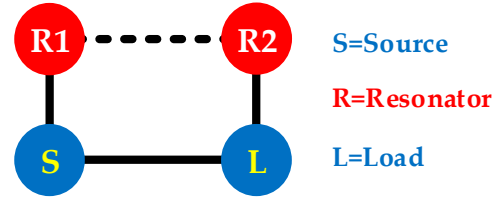

(b)

Figure 5. Configuration and coupling scheme of the proposed single-ended bandpass filter: (a) Detailed configuration; (b) coupling scheme.

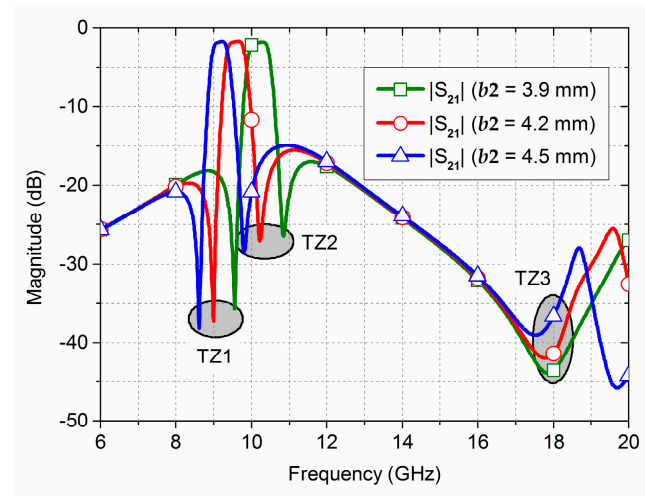

(a)

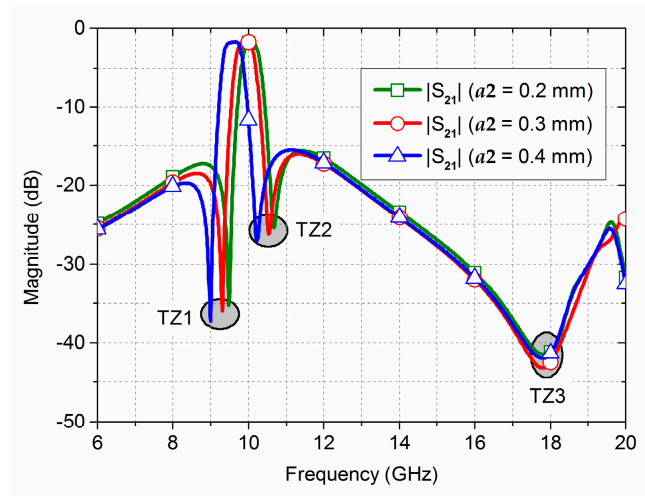

(c)

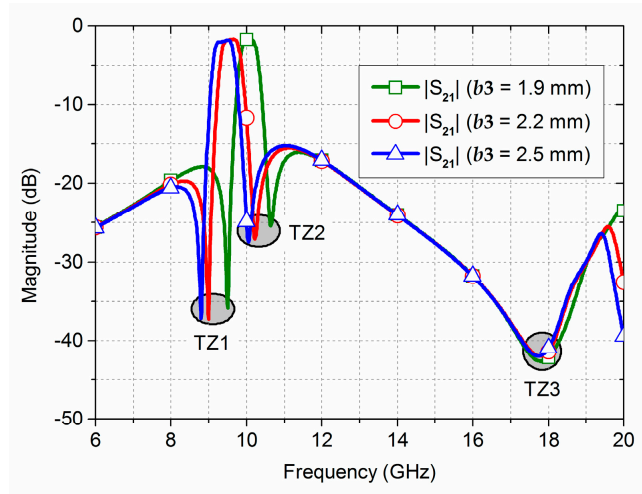

(b)

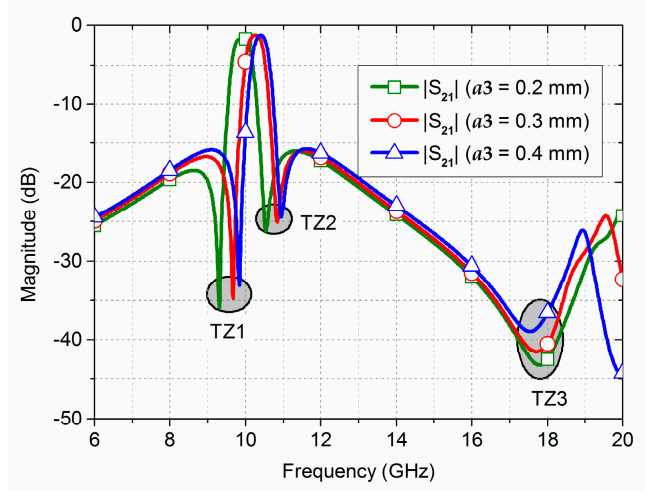

(d)

Figure 6. Simulated IS21 I of the proposed single-ended bandpass filter with the variation of some geometrical parameters: (a) $b 2$; (b) $b 3$; (c) $a 2$; (d) $a 3$.

The proposed single-ended filter in Figure 5a is designed with a central frequency of $10 \mathrm{GHz}$, a bandwidth of $70 \mathrm{MHz}$ (corresponding to a fractional bandwidth (FBW) of $7 \%$ ) and a ripple in passband of $0.1 \mathrm{~dB}$. With the aforementioned analyses, the filter is modeled and simulated by using the simulator mentioned above, and the optimized dimensions are listed as below: $w s=1.52, l t=1.8$, $a 0=1.0, b 0=2.0, a 1=0.4, b 1=6.0, c 1=2.0, v 1=0.4, s 1=0.54, s 2=0.25, s 3=0.35, a 2=0.36$, $a 3=0.2, b 2=4.2, b 3=2.1$ (units: $\mathrm{mm}$ ). Afterwards, the optimized single-ended filter is fabricated on a 
RT/Duroid 5880 substrate with a thickness of $0.508 \mathrm{~mm}$, a relative permittivity of $2.2 \pm 0.2$, and a loss tangent of 0.0009 . The fabrication is carried out by utilizing the standard printed circuits board (PCB) process, with a $2 \mu \mathrm{m}$-thick gold cover being electroplated on the copper metal after fabrication. The fabricated single-ended filter is measured by using a Keysight N5245A vector network analyzer and a test fixture. Figure 7 shows photographs and measured results of the fabricated single-ended filter. It can be seen in Figure 7a that the fabricated single-ended filter has a physical size of $15 \mathrm{~mm} \times 3.7 \mathrm{~mm}$, corresponding to an electrical size of $0.135 \lambda_{\mathrm{g}}{ }^{2}$, where $\lambda_{\mathrm{g}}$ is the guided wavelength of the microstrip line at the central frequency. According to Figure $7 \mathrm{~b}$, the fabricated single-ended filter achieves a central frequency of $10.12 \mathrm{GHz}$, a FBW of $6.5 \%$, an in-band insertion loss of $1.4 \mathrm{~dB}$, and a return loss better than $22 \mathrm{~dB}$. Moreover, three TZs, at $8.8 \mathrm{GHz}, 11.2 \mathrm{GHz}$, and $19 \mathrm{GHz}$, respectively, are achieved as well. Furthermore, as shown in Figure $7 c$, the fabricated single-ended filter exhibits a stopband covering $11 \mathrm{GHz}$ to $35 \mathrm{GHz}$ with rejection over $21 \mathrm{dBc}$, illustrating good stopband performance. Finally, from Figure 7d, the simulated group delay of S21 is less than $0.75 \mathrm{~ns}$, with a small in-band variation less than $0.2 \mathrm{~ns}$. Meanwhile, the measured group delay of S21 is less than $1 \mathrm{~ns}$, and the variation of group delay in the passband is less than $0.35 \mathrm{~ns}$, which is suitable for linear phase applications. Above all, the fabricated single-ended filter achieves selectivity, stopband and phase performance, and its measured results are in good agreement with the simulated ones.

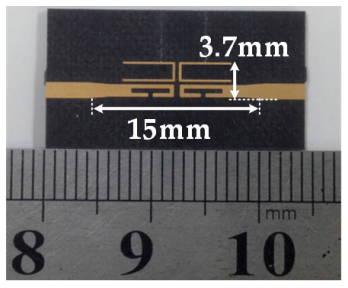

(a)

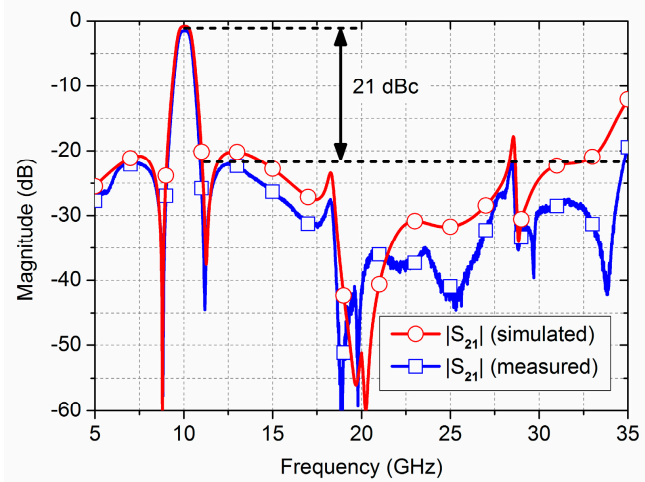

(c)

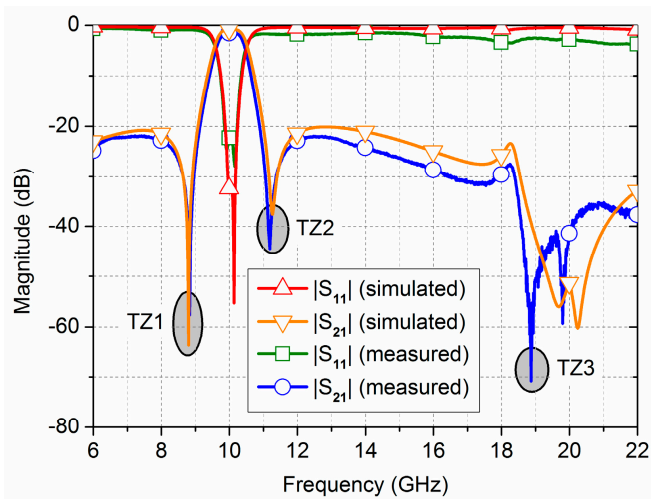

(b)

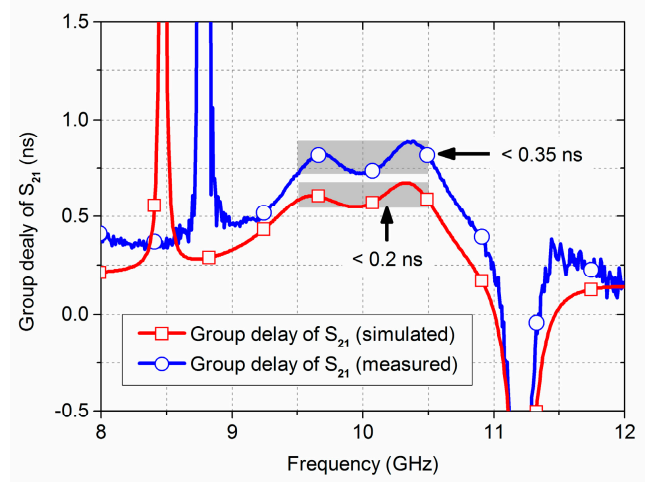

(d)

Figure 7. Photograph and measured results of the fabricated single-ended filter: (a) Photograph; (b) measured and simulated results; (c) wideband response of $\left|S_{21}\right|$; (d) measured group delay.

\section{Balanced Bandpass Filters with Source-Load-Coupled Spurlines}

It is well known that the differential transmission is better than the single-ended case, since the balanced signals are able to exhibit much better performance, including better immunity to environmental noise, weaker electromagnetic interference, better electromagnetic compatibility, and 
larger dynamic range [20]. Due to these advantages, balanced circuits and components have been widely used in wireless communication and sensing applications. For instance, in the fifth generation (5G) mobile communication system, the operating frequency becomes higher and its corresponding bandwidth gets wider as well. Consequently, circuits and components in the 5G system will be more sensitive to environmental noises. Hence, balanced components including filters are in urgent demand in wireless communication and sensing communities.

In order to further explore the availability of the USIR and the proposed T-shaped spurline in complex or multi-port microwave components, a balanced bandpass filter is designed. Figure 8 shows the detailed configuration and coupling scheme of the proposed balanced filter. As shown in Figure 8a, the proposed balanced filter consists of two USIRs, four T-shaped spurlines, and four $50 \mathrm{ohm}$ microstrip lines at the input and output ports. Moreover, it can be seen from Figure $8 \mathrm{~b}$ that there are four individual coupling paths (i.e., S-R1-R2-L, S-L, S'-R1-R2-L', and $S^{\prime}-\mathrm{L}^{\prime}$ ) in the proposed balanced filter. Among these coupling paths, S-R1-R2-L and $S^{\prime}-R 1-R 2-L^{\prime}$ are the major paths for signal transmission, while S-L and $S^{\prime}-L^{\prime}$ are the source-load coupling paths for TZ generation. More specifically, once the proposed balanced filter is in operation, coupling paths S-R1-R2-L and S-L can cross-couple to generate two TZs at the lower and upper sides of the passband. Meanwhile, the other two coupling paths, $\mathrm{S}^{\prime}-\mathrm{R} 1-\mathrm{R} 2-\mathrm{L}^{\prime}$ and $\mathrm{S}^{\prime}-\mathrm{L}^{\prime}$, will function in a similar way. Nevertheless, this does not mean that these four coupling paths can help to produce four TZs for the proposed balanced filter. In fact, although these four coupling paths can form two individual cross-couplings, they perform for different sides of the differential signal. Specifically speaking, as S-R1-R2-L and S-L form cross-coupling to produce two TZs for the transmission of the positive (or negative) side of the differential signal, $S^{\prime}-R 1-R 2-L^{\prime}$ and $S^{\prime}-L^{\prime}$ will operate in a similar way for the transmission of the negative (or positive) side of differential signal. In addition, T-shaped spurlines at the source and load nodes of the proposed balanced filter can help to further enhance its stopband performance. In practical operation, T-shaped spurlines at the $S$ and $L$ nodes can produce an extra $\mathrm{TZ}$ for the transmission of the positive (or negative) side of differential signal, while T-shaped spurlines at the $S^{\prime}$ and $L^{\prime}$ nodes are beneficial for the negative (or positive) side. Comparing Figure 5 with Figure 8, it can be seen that the proposed balanced filter can be regarded as a combination of the single-ended filter and its mirror duplicate.

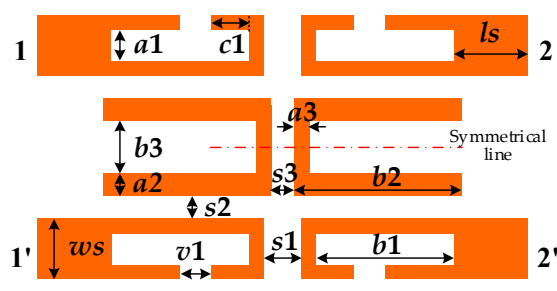

(a)

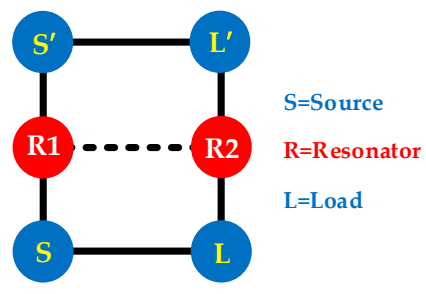

(b)

Figure 8. Configuration and coupling scheme of the proposed balanced filter: (a) Detailed configuration; (b) coupling scheme.

Before the balanced filter design, it is essential to investigate the influence of its constituent elements on its transmission properties. Influence from the USIR has been discussed in the realization of the single-ended filter; this can be applied to the balanced filter as well. This is mainly because the single-ended and balanced filters are with the same basic resonator and fundamental coupling scheme. Here, some numerical simulations have been carried out to study the influence of the T-shaped spurline on the transmission response of the balanced filter. In these simulations, the same commercial electromagnetic simulator as mentioned above is used, and the substrate is set with a thickness of $0.508 \mathrm{~mm}$, a relative permittivity of 2.2, a loss tangent of 0.001 , and a copper cover of $0.035 \mathrm{~mm}$. Some geometrical parameters are fixed as well: $w s=1.52, l s=3, s 1=0.6, s 2=0.5, s 3=0.8, a 2=0.4, a 3=0.2, b 2=4$, $b 3=2.2$ (units: $\mathrm{mm}$ ). Figure 9 shows simulated $|\mathrm{S} 21|$ under differential mode (DM) operation with 
the variations of various geometrical parameters of the T-shaped spurline. For Figure $9 \mathrm{a}, a 1=0.7 \mathrm{~mm}$, $b 1=3.7 \mathrm{~mm}, c 1=1 \mathrm{~mm}$. In this case, as $v 1$ becomes larger, the passband, TZ1, and TZ2 almost stay the same, while TZ3 moves to higher frequency. For Figure $9 b, a 1=0.7 \mathrm{~mm}, b 1=3.7 \mathrm{~mm}, v 1=0.3 \mathrm{~mm}$. In this case, with a larger $c 1, \mathrm{TZ3}$ also shifts higher, whereas the passband, TZ1, and TZ2 remain nearly unchanged. Moreover, the variation of $c 1$ will also have influence on the stopband performance. For Figure $9 \mathrm{c}, a 1=0.7 \mathrm{~mm}, c 1=1 \mathrm{~mm}, v 1=0.3 \mathrm{~mm}$. In this case, the passband, TZ1, and TZ2 just have very slight variety with the increasing of $b 1$, while TZ3 shifts notably lower. Finally, for Figure $6 \mathrm{~d}$, $b 1=3.7 \mathrm{~mm}, c 1=1 \mathrm{~mm}, v 1=0.3 \mathrm{~mm}$. In this case, the variation of $a 1$ only has a slight influence on the passband, TZ1, TZ2, and TZ3. It can be derived from Figure 9 that TZ3 is contributed to by the spurline, while TZ1 and TZ2 are produced by the cross-coupling attributed to the source-load coupling scheme.

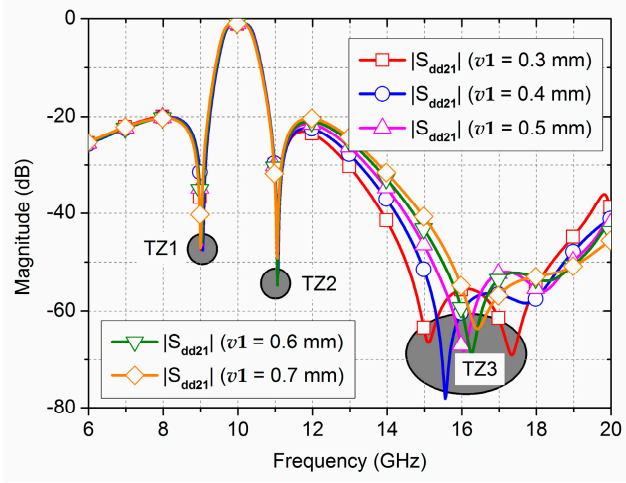

(a)

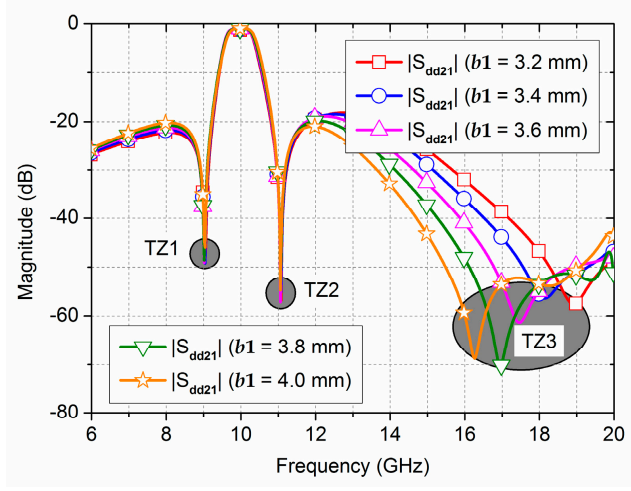

(c)

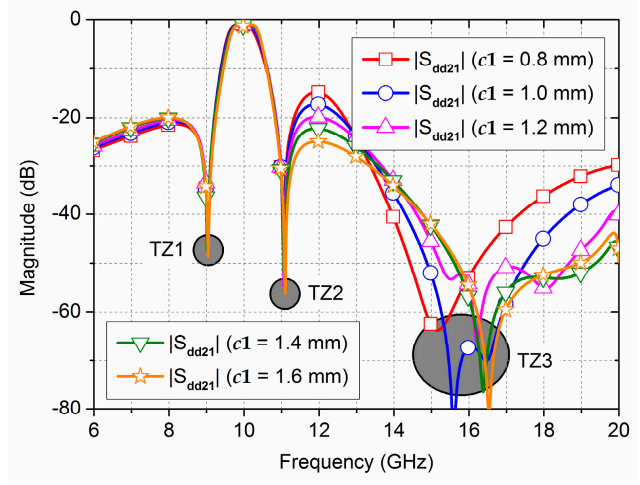

(b)

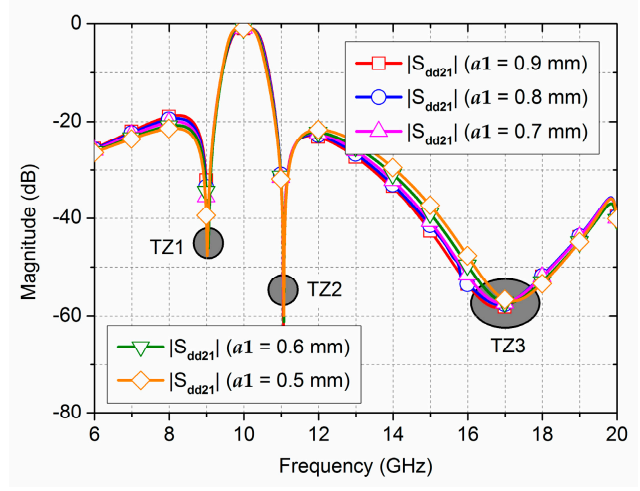

(d)

Figure 9. Simulated results of the proposed balanced filter with the variations of various geometrical parameters of the T-shaped spurlines: (a) $v 1$; (b) $c 1$; (c) $b 1$; (d) $a 1$.

Afterwards, the proposed balanced filter in Figure 8a is designed with the same specification parameters as the single-ended one (i.e., a central frequency of $10 \mathrm{GHz}$ and a FBW of $7 \%$ ). By using the same simulators mentioned above, its geometrical parameters are optimized to be: $w s=1.52$, $l s=1.0, a 1=0.72, b 1=3.8, c 1=1.35, v 1=0.37, s 1=0.65, s 2=0.51, s 3=0.62, a 2=0.4, b 2=4.3, a 3=0.2$, $b 3=2.1$ (units: $\mathrm{mm}$ ). The optimized balanced filter is fabricated by using the standard PCB process on a commercial Rogers RT/Duroid 5880 substrate with a relative permittivity of $2.2 \pm 0.02$, a relative permeability of 1 , a loss tangent of 0.0009 , and a thickness of $0.508 \mathrm{~mm}$. The measurement is carried out by using a Keysight N5245A vector network analyzer and a self-designed test fixture. Measured results of the fabricated BPF is illustrated in Figure 10, in which it can be seen easily that the measured results agree with the simulated ones well. According to Figure 10a, the prototyped balanced filter achieves a $1 \mathrm{~dB}$ fractional bandwidth about $6.1 \%$, a minimum in-band insertion loss of $0.9 \mathrm{~dB}$, and three $\mathrm{TZs}$ 
located at $9 \mathrm{GHz}, 11 \mathrm{GHz}$, and $15.2 \mathrm{GHz}$, all under the DM operation. From the wideband response of IS21 I under the DM operation given in Figure 10b, its upper stopband is far from up to $40 \mathrm{GHz}$ (i.e., the fourth harmonic) with the suppression better than $20 \mathrm{~dB}$. The measured in-band absolute group delay of S21 under the DM operation is less than $1.3 \mathrm{~ns}$, with a small variation less than $0.3 \mathrm{~ns}$, as shown in Figure 10c. It can be captured easily from Figure 10c that the measured in-band absolute group delay of S21 is about 0.25 ns larger than the simulated one, whereas the measured variation is nearly as same as the simulation. Such group delay performance illustrates that the fabricated balanced filter is suitable for digital data transmission applications in communication systems. Moreover, according to the results under the $\mathrm{CM}$ operation in Figure 10d, it can be seen that the measured insertion loss under the CM operation is over $17 \mathrm{~dB}$, which means the measured CMRR is better than $16.1 \mathrm{~dB}$. Additionally, it can be obtained easily from Figure 10e that the functional part of the fabricated balanced filter has a physical size of $9.4 \mathrm{~mm} \times 5.7 \mathrm{~mm}$, corresponding to an electrical size of $0.129 \lambda_{\mathrm{g}}{ }^{2}$.

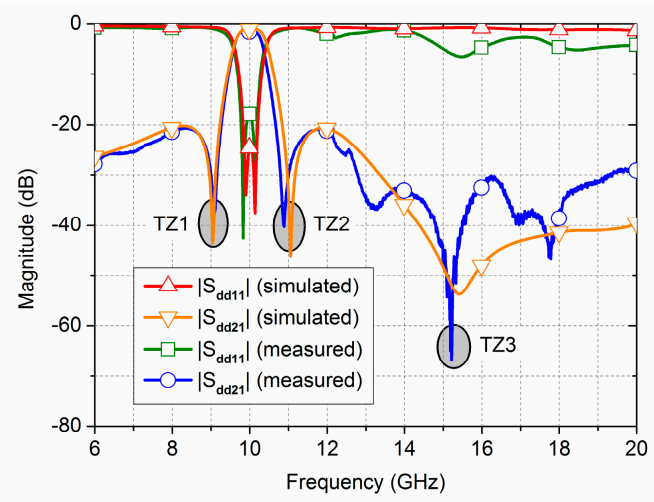

(a)

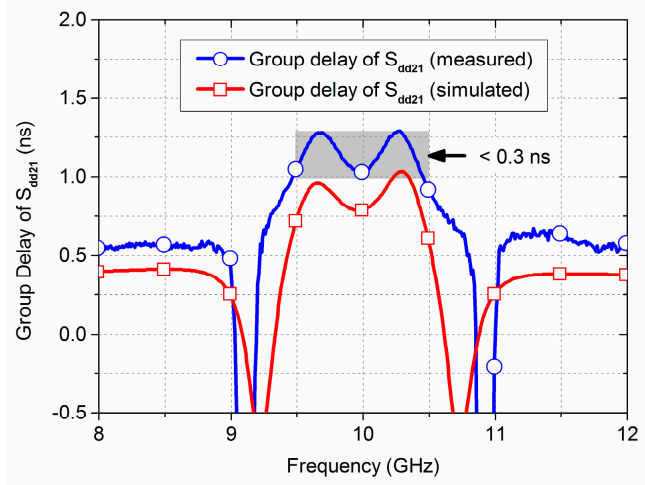

(c)

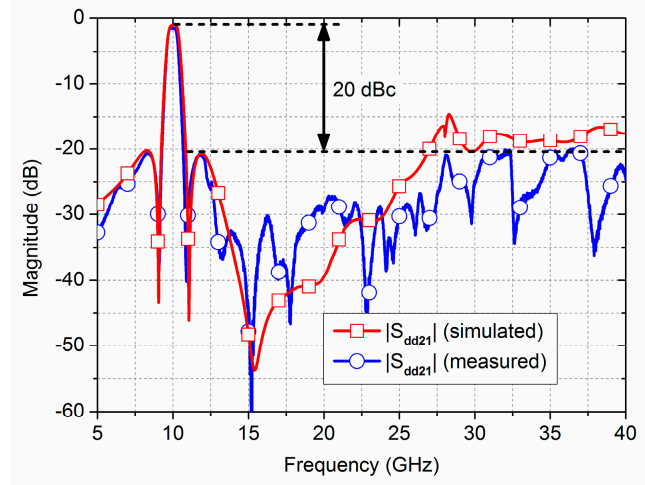

(b)

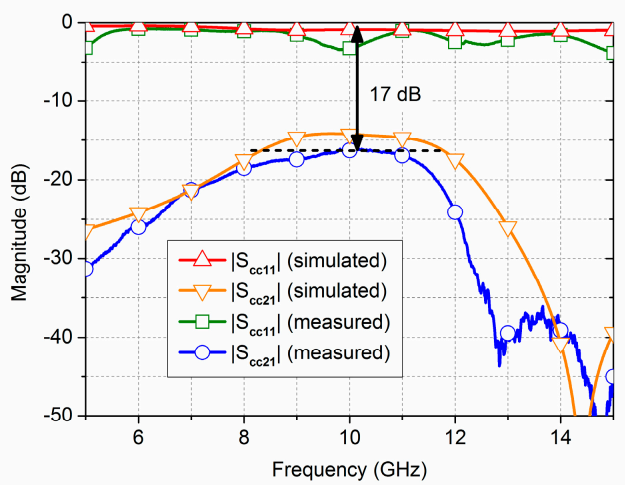

(d)

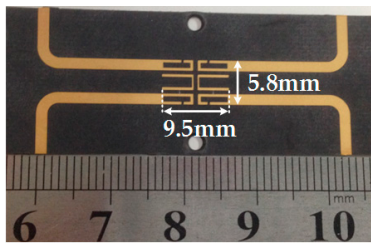

(e)

Figure 10. Measured results and photograph of the fabricated balanced filter: (a) IS21 I and IS11 I under differential mode (DM) operation; (b) wideband response of IS21 I under DM operation; (c) IS21 I and IS11 | under CM operation; (d) measured group delay of S21 under DM operation; (e) photograph. 


\section{Discussion}

Table 1 summarizes the comparison between the proposed balanced filter and some similar reported works. As compared with the works reported in $[11,13,15]$, the proposed one has similar insertion loss and the largest number of TZs, which means the proposed one exhibits better selectivity performance. Moreover, the proposed balanced filter has wider a stopband, with about $20 \mathrm{~dB}$ out-of-band rejection as well. Even though the previously reported balanced filters listed in Table 1 have higher CMRR than the proposed one, they also suffer the issue of larger electrical sizes. For instance, compared with the balanced filter reported in [15], the proposed one achieves an electrical size reduction of approximately half. In addition, the differential filter reported in [13] is very unique since it utilizes lumped resistors in the filter to absorb CM noise. Hence, in the passband of this differential filter, both the transmission coefficient and reflection coefficient under the CM operation (i.e., $S_{\mathrm{cc} 21}$ and $\mathrm{S}_{\mathrm{cc} 11}$ ) have low values. More specifically, as reported in [13], the measured $\left|S_{\mathrm{cc} 11}\right|$ in the passband is about $-25.38 \mathrm{~dB}$, while the measured $\left|S_{\mathrm{cc} 21}\right|$ in the passband is slightly lower than the $\left|S_{\mathrm{cc} 11}\right|$. Meanwhile, the measured insertion loss under the DM operation is as small as $0.29 \mathrm{~dB}$. Therefore, it can be obtained that the differential filter in [13] achieved a CMRR around $25 \mathrm{~dB}$. It can be seen from Table 1 that the proposed balanced filter has the best selectivity and out-of-band rejection and the smallest electrical size.

Table 1. Comparison between the proposed balanced filter and some similar reported works.

\begin{tabular}{|c|c|c|c|c|c|c|}
\hline Ref. & $\begin{array}{l}f_{0}, \mathrm{FBW}^{1} \\
(\mathrm{GHz}, \%)\end{array}$ & $\begin{array}{l}\text { Insertion } \\
\text { Loss (dB) }\end{array}$ & $\begin{array}{l}\text { Number } \\
\text { of } \mathrm{TZ}^{2}\end{array}$ & $\begin{array}{l}\text { Stopband } \\
\left(\mathrm{dBc}, n \cdot f_{0}\right)\end{array}$ & $\begin{array}{c}\text { CMRR }^{3} \\
(\mathrm{~dB})\end{array}$ & $\begin{array}{l}\text { Electrical Size } \\
\quad\left(\lambda_{0}^{2} / \varepsilon_{r}\right)\end{array}$ \\
\hline [11] & 4,16 & 1.5 & 0 & 20,2 & 30 & 0.510 \\
\hline [13] & $2,55.5$ & 0.29 & 1 & N.A. & 25 & 0.329 \\
\hline [15] & $1.9,47.4$ & 1.5 & 2 & $22,3.4$ & 28 & 0.251 \\
\hline Ours & $10,6.1$ & 0.9 & 3 & 20,4 & 16.1 & 0.128 \\
\hline
\end{tabular}

${ }^{1}$ FBW: fractional bandwidth. ${ }^{2} \mathrm{TZ}$ : transmission zero. ${ }^{3}$ CMRR: common-mode rejection ratio.

\section{Conclusions}

USIR-based single-ended and balanced bandpass filters with compact transverse sizes, high selectivity, and wide stopbands were presented. Particularly, the balanced filter has exhibited good DM and CM performance, which is jointly attributed to the source-load-coupled spurlines and USIRs. Meanwhile, the out-of-band rejection over $20 \mathrm{~dB}$ under DM operation of the balanced filter was extended up to the fourth-order harmonic of the central frequency, with its CMRR being better than $16.1 \mathrm{~dB}$ through the passband as well. These results indicate that the proposed balanced filter is a promising alternative for microwave and millimeter circuit and system applications.

Author Contributions: Conceptualization, F.Y. and K.W.; investigation, Y.M.H. and T.H.; writing-original draft preparation, F.Y. and Y.M.H.; writing—review and editing, S.D. and M.B.

Funding: This research was funded by National Natural Science Foundation of China, grant no. 61601087; Sichuan Science and Technology Program, grant no. 2018GZ0518.

Conflicts of Interest: The authors declare no conflict of interest.

\section{References}

1. Rebeiz, G.M.; Entesari, K.; Reines, I.C.; Park, S.J.; Eltanani, M.A.; Grichener, A.; Brown, A.P. Tuning in to RF MEMS. IEEE Microw. Mag. 2009, 10, 55-72. [CrossRef]

2. Pozar, D.M. Microwave Engineering, 4th ed.; Wiley: New York, NY, USA, 2012; pp. 48-89. ISBN 978-0-470-63155-3.

3. Makimoto, M.; Yamashita, S. Bandpass filter using parallel coupled stripline stepped impedance resonators. IEEE Trans. Microw. Theory Tech. 1980, 28, 1413-1417. [CrossRef] 
4. Pinel, S.; Bairavasubramanian, R.; Laskar, J.; Papapolymerou, J. Compact planar and vialess composite low-pass filters using folded stepped-impedance resonator on liquid-crystal-polymer substrate. IEEE Trans. Microw. Theory Tech. 2005, 53, 1707-1712. [CrossRef]

5. Roychoudhury, S.; Parui, S.K.; Das, S. Improvement of stopband using a spiral defected microstrip and defected ground structures. In Proceedings of the IEEE Applied Electromagnetics Conference, Kolkata, India, 18-22 December 2011.

6. Liu, H.; Knoechel, R.H.; Schuenemann, K.F. Miniaturized bandstop filter using meander spurline and capacitively loaded stubds. ETRI J. 2007, 29, 614-618. [CrossRef]

7. Liao, C.K.; Chang, C.Y. Design of microstrip quadruplet filters with source-load coupling. IEEE Trans. Microw. Theory Tech. 2005, 53, 2302-2308. [CrossRef]

8. Liu, H.; Liu, F.; Guan, X.; Ren, B.; Liu, T.; Wang, Y.; Xu, H. Wide-stopband superconducting bandpass filter using slitted stepped-impedance resonator and composite spurline structure. IEEE Trans. Appl. Supercond. 2018, 28, 1501508. [CrossRef]

9. Ponchak, G.E. Coplanar stripline spurline stub resonators with even-mode suppression for bandpass and bandstop filters. IEEE Microw. Wirel. Compon. Lett. 2018, 28, 1098-1100. [CrossRef]

10. Shi, J.; Xue, Q. Balanced bandpass filters using center-loaded half-wavelength resonator. IEEE Trans. Microw. Theory Tech. 2010, 58, 970-977.

11. Fernandez-Prieto, A.; Qian, S.L.; Hong, J.; Martel, J.F.; Medina, F.; Mesa, F.; Naqui, J.; Martin, F. Commonmode suppression for balanced bandpass filters in multilayer liquid crystal polymer technology. IET Microw. Antennas Propag. 2015, 9, 1249-1253. [CrossRef]

12. Sans, M.; Selga, J.; Velez, P.; Rodriguez, A.; Bonanche, J.; Borria, V.E.; Martin, F. Automated design of common-mode suppressed balanced wideband bandpass filters by means of aggressive space mapping. IEEE Trans. Microw. Theory Tech. 2015, 63, 3896-3908. [CrossRef]

13. Zhang, W.; Wu, Y.; Liu, Y.; Yu, C.; Hasan, A.; Ghannouchi, F.M. Planar wideband differential-mode bandpass filter with common-mode noise absorption. IEEE Microw. Wirel. Compon. Lett. 2017, 27, 458-460. [CrossRef]

14. Ebrahimi, A.; Baum, T.; Ghorbani, K. Differential bandpass filters based on dumbbell-shaped defected ground resonators. IEEE Microw. Wirel. Compon. Lett. 2018, 28, 129-131. [CrossRef]

15. Sans, M.; Selga, J.; Velez, P.; Bonanche, J.; Rodriguez, A.; Borria, V.E.; Martin, F. Compact wideband balanced bandpass filters with very broad common-mode and differential-mode stopbands. IEEE Trans. Microw. Theory Tech. 2018, 66, 737-750. [CrossRef]

16. Fernandez-Prieto, A.; Lujiambio, A.; Martel, J.; Medina, F.; Mesa, F.; Boix, R.R. Balanced-to-balanced microstrip diplexer based on magnetically coupled resonators. IEEE Access 2018, 6, 18536-18547. [CrossRef]

17. Gomez-Garcia, R.; Munoz-Ferreras, J.M.; Feng, W.; Psychogiou, D. Balanced symmetrical quasi-reflectionless single- and dual-band bandpass filters. IEEE Microw. Wirel. Compon. Lett. 2018, 28, 798-800. [CrossRef]

18. Feng, W.; Zhao, Y.; Che, W.; Gomez-Garcia, R.; Xue, Q. Single-ended-to-balanced filtering power dividers with wideband common-mode suppression. IEEE Trans. Microw. Theory Tech. 2018, 66, 5531-5542. [CrossRef]

19. Sagawa, M.; Makimoto, M.; Yamashita, S. Geometrical structure and fundamental characteristics of microwave stepped-impedance resonators. IEEE Trans. Microw. Theory Tech. 1997, 45, 1078-1085. [CrossRef]

20. Ting, H.-L.; Hsu, S.-K.; Wu, T.-L. Broadband eight-port forward-wave directional couplers and four-way differential phase shifter. IEEE Trans. Microw. Theory Tech. 2018, 66, 2161-2169. [CrossRef]

(C) 2019 by the authors. Licensee MDPI, Basel, Switzerland. This article is an open access article distributed under the terms and conditions of the Creative Commons Attribution (CC BY) license (http:/ / creativecommons.org/licenses/by/4.0/). 